\title{
A Radiological Method of Predicting Clinical Outcomes in PCL Reconstruction
}

\author{
Deborah Miao'en Huang*, Mohammed Tauheed and Denny Lie Tijauw Tjoen \\ Department of Orthopaedic Surgery, Singapore General Hospital, Singapore
}

\begin{abstract}
Aim: This study evaluates the feasibility of using radiographs as a means to predict the clinical outcome of $\mathrm{PCL}$ reconstruction surgery.

Material and methods: 37 patients who had undergone PCL reconstruction between 2009 and 2014 were included. A standard follow-up was performed for at least two years and consisted of pre- and post-op Lysholm scores. Lateral knee radiographs were studied and the measurements of the tibial Plateau to tibial Tunnel Length (PTL) and superior tip of Fibula to tibial Tunnel Length (FTL) were collected. A correlation was then derived and illustrated via scatter plots.

Results: 30 out of the 37 patients who responded to the follow-up phone interview had a mean Lysholm score of 86.34 ( \pm 9.6). The measurements for above mentioned lengths PTL and FTL averaged $15.54 \pm 5.15 \mathrm{~mm}$ and $10.32 \pm 4.84 \mathrm{~mm}$ respectively. There was a good positive correlation between PTL and improvement in Lysholm scores (0.78) and a good negative correlation between FTL and improvement in Lysholm scores (-0.92).

Conclusion: We report our finding of a correlation between position of tibial tunnel with respect to superior tip of fibula, and clinical outcomes for PCL reconstruction. Our data suggests that better clinical outcomes are achieved when the tibial tunnel exit point is closer to the superior tip of the fibula. We propose the use of this radiological method of measurement (both intra- and post-operatively) as a possible means to predict the clinical outcomes of PCL reconstruction surgery.
\end{abstract}

\section{Keywords}

PCL reconstruction, Lyshom score, Tibial tunnel, Lateral knee radiographs

\section{Introduction}

The posterior cruciate ligament $(P C L)$ is not as well studied as the anterior cruciate ligament $(\mathrm{ACL})$ because the incidence of acute PCL injuries is low at $3.4 \%$ to $20 \%$ of all knee injuries ([1] as cited in [2]). This leads to many differing views towards its management $[3,4]$.

The focus of our study is on the tibial attachment of the PCL. This has been described to be below the joint line and over the back of the tibial plateau in the posterior half of the PCL facet (posterior "central depression between the medial and lateral portion of tibial plateau") [5], and it has been shown that the working fibers of the PCL is situated " $7 \mathrm{~mm}$ anterior to the posterior cortex of the tibia, measured along the PCL facet" [5]. Since there is consensus or clinical evidence for an exact tibial tunnel placement, it is recommended that tunnel placement of PCL should follow its original anatomy [6].

The goal of our study was to look at possible radiological method to predict outcome of PCL reconstruction. We looked into the probable correlation between the subjective outcomes of patients using the Lysholm score with the exit site of the graft from the tibia. We then calculated and looked at the following measurements 1 ) The distance of the top of tibial to the tibial graft exit site and 2) Head of fibula with the graft exit site. Most of the studies take the posterior slope of the tibial plateau or the joint line as a reference for the $P C L$ exit point, however we are the first to have taken the superior tip of the fibula as our reference point for tibial exit of PCL.

\section{Materials and Methods}

In a retrospective single-surgeon study from 2009 to 2014, patients who had undergone $\mathrm{PCL}$ reconstruction had their clinical data collected. Diagnosis was made via clinical examination and MRI scans. Clinical examination included a posterior sag sign, loss of normal tibial step-off (grade 3), posterior

*Corresponding author: Deborah Miao'en Huang, Department of Orthopaedic Surgery, Singapore General Hospital, 20 College Road, Academia, Level 4, 169856, Singapore

Accepted: June 12, 2019

Published online: June 14, 2019

Citation: Huang DM, Tauheed M, Tjoen DLT (2019) A Radiological Method of Predicting Clinical Outcomes in PCL Reconstruction. Arch Sports Med 3(1):156-161 


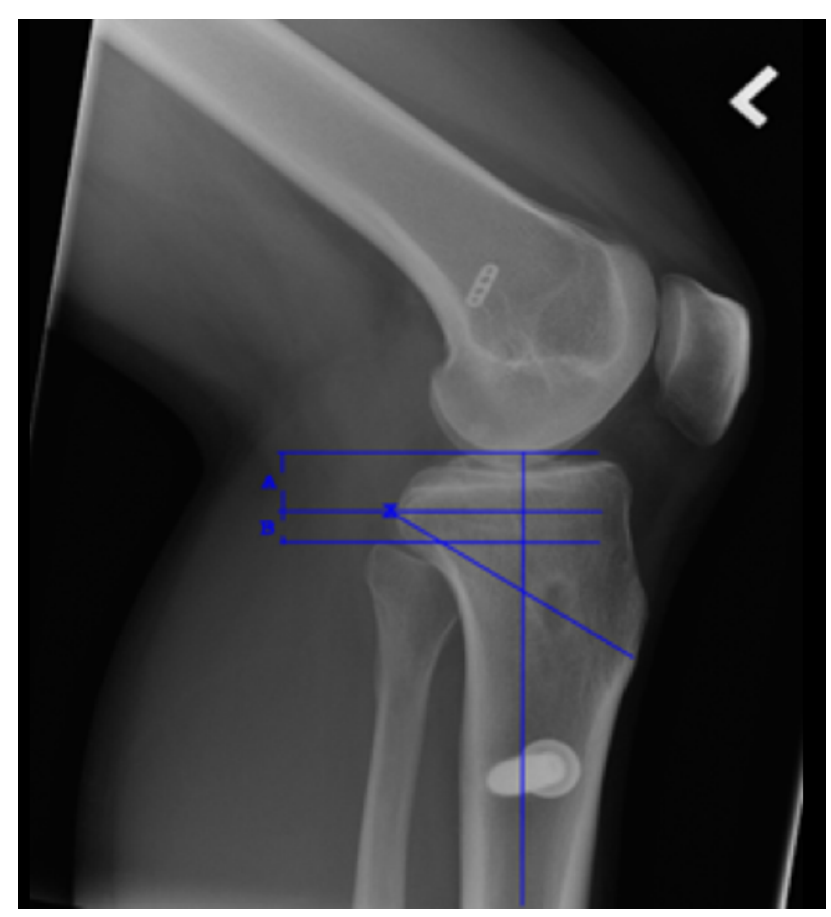

Figure 1: An example of measurements taken from a radiograph.

drawer and dial test. MRI scans then confirmed the PCL injury and whether there were other concomitant meniscus or ligamentous injury. Indication for surgery included patients that were symptomatic (pain and/or instability) despite a trial of 3 to 6 months of conservative treatment, and patients were wanted to return to their previous high level of sporting activity. The same method of PCL reconstruction was performed for all patients, with similar steps taken throughout by the same surgeon. Patients had radiographs of the knee done the day after surgery, which included a true lateral radiograph of the knee. The radiographers at our centre followed standard guidelines in order to obtain a true lateral image of the knee where there is superimposition of both distal femur condyles, slight superimposition of the fibular head with the tibia and an open patellofemoral joint space. Out of 37 cases, only 30 patients completed at least two years of follow-up and were included. 7 patients were lost to follow-up or had incomplete data. These 30 patients were interviewed and pre- and post-operative Lysholm scores were obtained.

\section{Measurements}

Radiographs were taken on the second post-operative day and these radiographs were obtained from the hospital's picture archiving and communication system (PACS). The procedures for collection of the required measurements are as follows. First, a line is drawn along the longitudinal axis of the tibia. Next, a line is drawn in the centre of the tibial tunnel, with point $X$ being the posterior exit site of the tibial tunnel (Point $X$ in Figure 1 ). With Point $X$ as a reference, another line is added at right angles to the longitudinal tibial axis line. The last 2 lines drawn are also perpendicular to the longitudinal tibial axis and parallel to the line through Point $X$ - a superior line along the highest point of the tibia plateau and an inferior line from the tip of the fibular head. Distances are now measured between Point $\mathrm{X}$ and the superior line (labeled as PTL - plateau-tunnel length) and between Point $X$ and the inferior line (labeled as FTL - fibular-tunnel length). PTL represents the distance from the tibial plateau to the midpoint of the tibial tunnel and FTL represents the distance between the midpoint of the tibial tunnel to the superior tip of the fibula. An illustration of the various lines drawn is included in Figure 1. PTL and FTL are referred to as Distances $A$ and $B$ respectively, and Point $X$ represents the midpoint of the tibial tunnel exit site in Figure 1.

\section{Operative technique}

Both semitendinosus and gracilis tendons were harvested and doubled upon itself by whip-stitching the free ends. Conventional medial para-patellar portals, an anterolateral portal and a high posteromedial portal, were used. With the camera in the anteromedial portal, the synovitis and remnant $\mathrm{PCL}$ in the anterior compartment were debrided. The posterior compartment was visualised and the footprint of the PCL was debrided using a shaver via the posteromedial portal. The capsule was carefully peeled away using both the shaver and radiofrequency wands. In order to prevent injury to the neurovascular structures which are in close proximity [7], the knee were flexed to 90-degrees and fluid pressure increased to 90 $\mathrm{mmHg}$ to allow posterior displacement of the joint capsule.

The posterior compartment of the knee is viewed with the camera in the posteromedial portal. A PCL guide is inserted through the medial para-patellar portal, past the intercondylar notch and positioned just lateral to the midline on the fossa for the PCL, about $10 \mathrm{~mm}$ below the visualised joint line. Under direct visualization, a guide pin is drilled until its tip emerges from the posterior tibial cortex. The tibial tunnel is reamed to size and a $10 \mathrm{Fr}$ Nelaton catheter is inserted into the tibial tunnel and retrieved through the anterolateral portal.

The centre of the femoral footprint is located $8 \mathrm{~mm}$ posterior to the articular margin and at a 10:30 position for the left knee and 1:30 position for the right knee. The footprint is debrided and a short Beath pin is drilled into the femoral footprint via the anterolateral portal, passing through the medial femoral cortex and exiting the skin anteromedially. A cannulated reamer is used to create a $35-\mathrm{mm}$ deep tunnel of the appropriate size. The free suture is passed through the Beath pin in the femoral tunnel and pulled out of the medial femoral condyle. The other end of the suture is looped to the Nelaton catheter and pulled out of the tibial tunnel. The graft is then passed through the loop of free suture emerging from the tibial tunnel. Under direct visualisation, the suture is pulled to pass the graft from distal to proximal until the graft is engaged into the femoral tunnel and the endobutton flips. An obturator, entering through the posteromedial portal, is used to reduce the acute angle of the killer turn by guiding the graft slightly more posteriorly as it exits the tibial tunnel to enter the femoral tunnel. Femoral fixation is achieved with both an endobutton and absorbable interference screw through the anterolateral portal with the knee at 100 degrees of flexion. The graft is cycled 20 times before the distal end is secured with an absorbable interference screw and tibial 
post. This is done with the knee in 70 degrees of flexion and with an anterior drawer force applied to restore the normal anterior tibial step-off.

\section{Results}

Age of patients ranged from 20 to 30 -years-old, with predominant right sided involvement (right side 22, left side 8 ). There were 22 males and 8 females. Most of the cases were isolated $P C L$ injuries $(n=23)$ treated with a single anterolateral bundle $\mathrm{PCL}$ reconstruction technique [8].

The diagnosis was made via clinical examination and MRI scans. All patients had clinical examination findings of a positive posterior sag sign, loss of normal tibial step-off (grade 3) and a positive posterior drawer test. MRI scans confirmed the $\mathrm{PCL}$ injury and the presence of other concomitant meniscus or ligamentous injury. All but one of the PCL tears were intrasubstance ruptures.
The diagnosis was made clinically and then confirmed on MRI scan as discussed above (in the methods section. 14 cases were acute PCL injuries, with 7 acute cases presenting with concomitant meniscus and other ligamentous injuries. Two of the patients had medial meniscal injury, of which one underwent meniscus repair, and the other that had a bucket handle tear underwent a partial medial meniscectomy. Five of the patients with lateral meniscal tears all underwent meniscal repair. The 7 patients with concomitant meniscus injuries were symptomatic (pain and instability) and require early surgery. The other 7 acute PCL injury patients were young patients who were symptomatic (pain and instability) from the isolated PCL injury and keen to return to pre-injury level of sporting activity.

16 cases were chronic isolated $\mathrm{PCL}$ injuries and these patients have undergone at least 3-6 months of conservative treatment before surgery was performed.

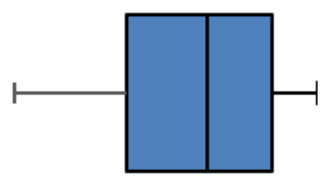

口 POST OP LYSHOLM

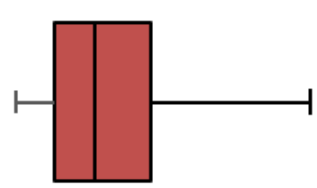

PRE OP LYSHOLM 20
40

60

80

Figure 2: Box plot of Pre- and Post-Operative Lysholm scores.
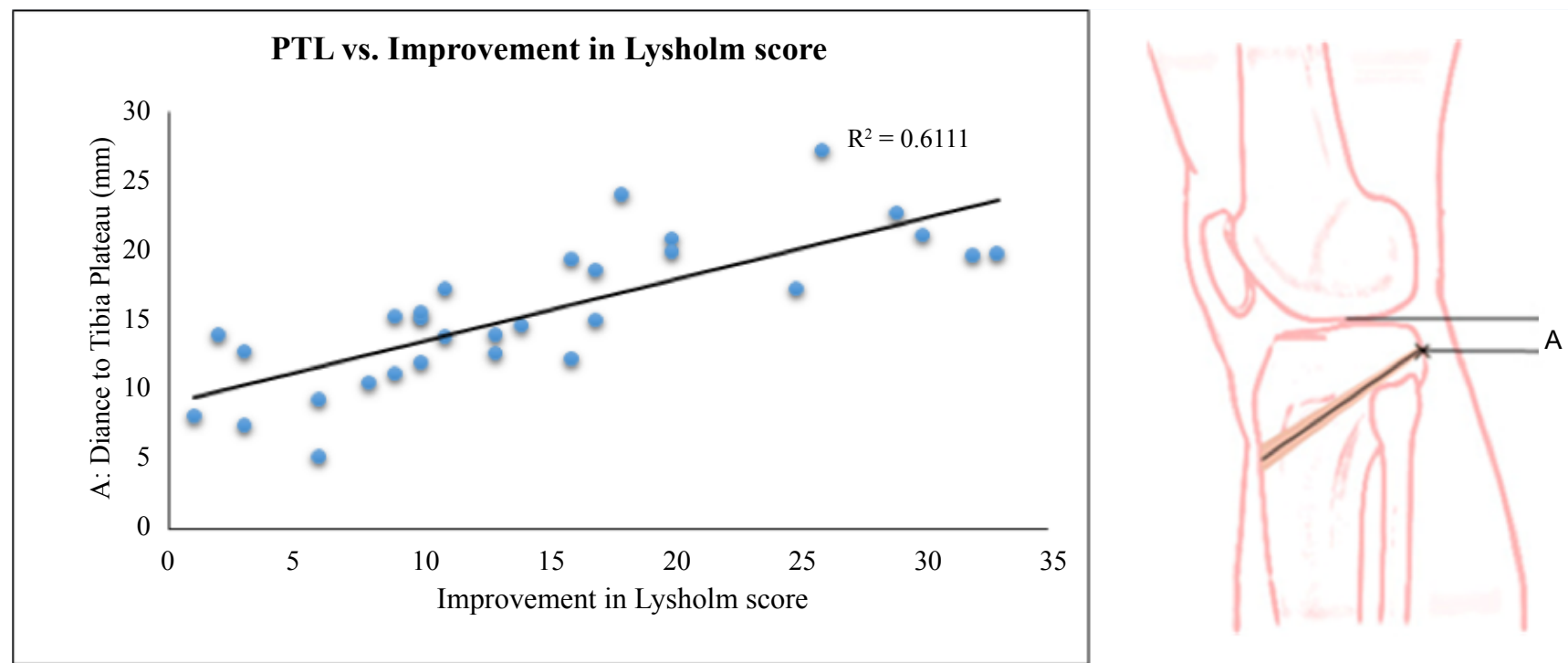

Figure 3: Plateau Tunnel Length as a function of improvement in Lysholm scores. 
The average pre-operative Lysholm knee score significantly improved $(p<0.01)$ from $71.73 \pm 7.8$ to post-operative average of $86.33 \pm 9.6$ at the final follow up (See Figure 2). The average measured PTL was $15.54 \pm 5.15 \mathrm{~mm}$ (ranging from 5.21 to $27.33 \mathrm{~mm}$ ), and the average measured FTL was 10.32 $\pm 4.84 \mathrm{~mm}$ (ranging from 2.25 to $18.94 \mathrm{~mm}$ ). We made use of scatter plots to better understand the relationship between improvement in Lysholm score, with respect to, both PTL (Figure 3) and FTL (Figure 4). The Pearson correlation coefficients for the relationship between improvement in Lysholm score versus PTL and FTL was 0.78 and -0.92 respectively. These values suggest that there is a correlation between tibial tunnel positioning and clinical outcomes for PCL reconstruction surgery. The further away the tibial tunnel exit point was from the tibial plateau and the closer this same point was to the superior tip of the fibula, the better the scores. This suggests a potential way of radiographically predicting better clinical results.

\section{Discussion}

The primary finding of this study is the presence of a correlation between clinical outcomes and the placement of the tibial tunnel, with respect to the superior tip of the fibula, in PCL reconstruction surgery. The finding is timely as there is a growing interest in the use of radiographic tools to assist in determining optimal tibial tunnel positions and then translating the outcomes into clinical practice $[6,9,10]$. To our best knowledge, this is the first time an objective investigation was conducted to determine whether a relationship exists between the placement of the tibial tunnel with respect to the fibula head, and clinical outcomes, and whether its placement can be used to predict outcomes.

Existing works suggest that overall surgical outcomes after isolated $\mathrm{PCL}$ reconstructions are inconsistent and less satisfactory than those of $A C L$ reconstructions [11]. One explanation is that this may be due to certain differences from the $\mathrm{ACL}$, namely, the more complex anatomy of the $\mathrm{PCL}$, the non-existence of an isometric point and a constant posterior gravity force on the PCL graft at rest [12]. In general, however, clinical subjective and objective outcomes usually improve post-PCL reconstruction. Our mean improvement of $14.6 \pm$ 9 points is consistent with similar studies that employ the single-bundle (SB) PCL reconstruction technique, where the mean improvement in Lysholm scores have been reported to range from 15.9 to 27.1 points. While our results may appear to lie in the lower end of this spectrum, this can possibly be accounted for by the few outliers in our small sample size. We also add that most studies also report an average of one grade of improvement in anteroposterior (AP) laxity postSB PCL reconstruction $[2,13,14]$, even though this might not completely restore knee kinematics $[13,15]$.

Our study was focused on isolated PCL injured knees that were treated using the single anterolateral (AL) bundle PCL reconstruction technique [8]. The AL bundle is significant because it represents two-thirds of PCL mass and is reconstructed in single-bundle techniques [8]. Although recent studies focussing on double-bundle reconstruction techniques appear to better restore knee kinematics [16-18], it is found that there are no significant clinical differences between single versus double-bundle techniques [8]. It is also found that there is no established clinical difference between the use of autograft versus allograft, transtibial tunnel versus tibial inlay techniques or remnant-preserving versus remnant-release techniques [8].

The anatomy and isometry of the PCL has been well studied and most of these studies show that the femoral attachment point is the crucial factor in obtaining $\mathrm{PCL}$ isometry [19], as compared to the less crucial tibial attachment point of the PCL $[19,20]$. This is because modifications in the tibia only produced minor changes in the posterior motion limits $[19,21]$ and causes small alterations in isometry, whereas changes in the localization of points in the femur can cause large variations in isometry [20,22-27]. Restoration of knee stability in flexion has been shown to be strongly dependent
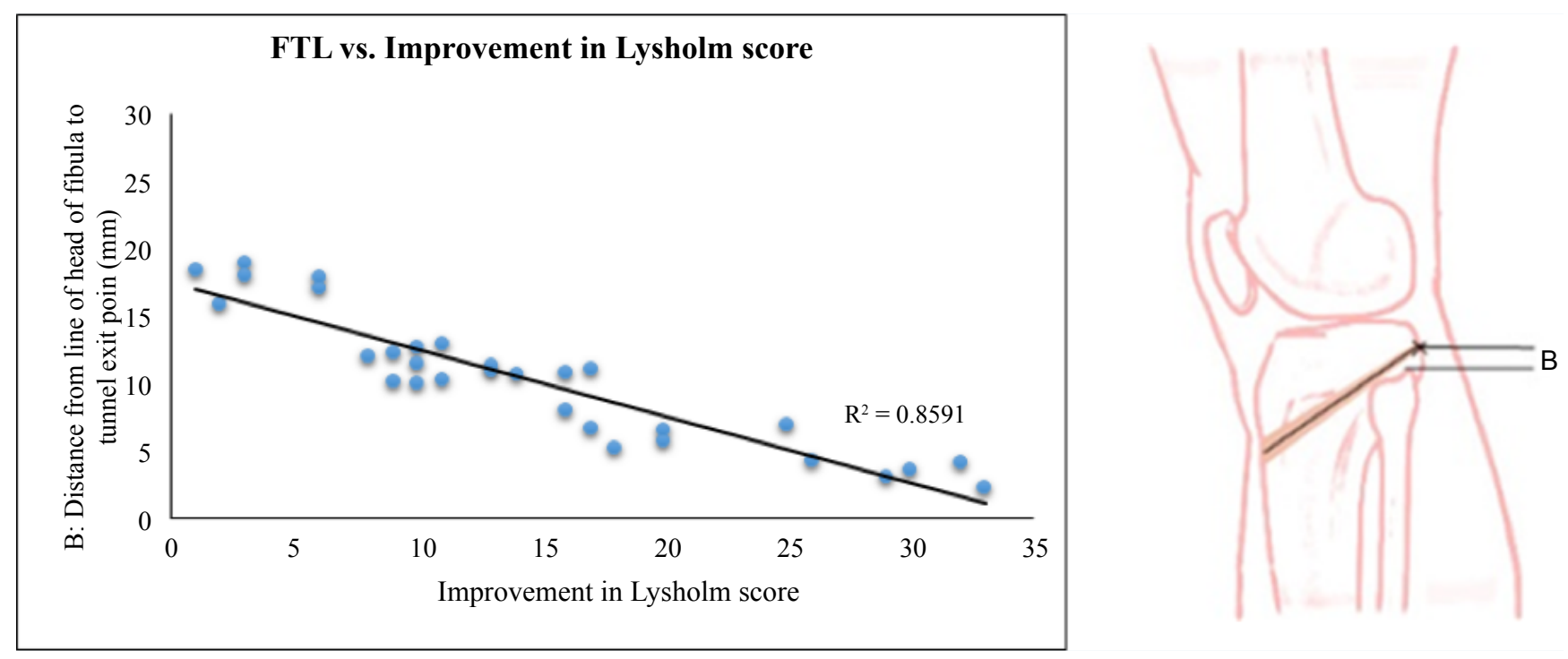

Figure 4: Fibula Tunnel Length as a function of improvement in Lysholm scores. 
on the femoral attachment location, particularly the selected proximal-distal location [28]. On top of this, isometric PCL reconstruction has been shown to be inferior in performance, when compared to a more anatomic tunnel placement in $\mathrm{PCL}$ reconstruction. This is attributed to the fact that isometric reconstruction causes over-constraint in extension and posterior translation laxity/instability in flexion $[19,28]$.

There is a growing interest in determining the best tibial tunnel position and the ability to translate that finding into clinical practice, with the aid of intraoperative radiographic guidance $[8,27,29]$. To our best knowledge, this current study is the first in an attempt to investigate if a trend/relationship exists between the position of the tibial tunnel and clinical outcomes, and whether placement is able to predict outcomes.

At the time of writing, in spite of the many studies since Girgis, Marshall's [30] analysis in 1975, there has been no consensus regarding the exit point of the tibial tunnel $[5,6,28,30-33]$. While many studies suggest that the placement of the tibial tunnel should be around 1 to 1.5 $\mathrm{cm}$ distal to the tibiofemoral joint line [6,34-36], it is known that this approach would not place the tunnel aperture in a perfectly anatomic position [33]. The fixed distance from the posterior tibial cortex or the posterior slope of tibial plateau to the exit point of PCL on the tibia is not a uniform length, especially between genders $[9,10]$. Medial-lateral tibial tunnel placement in PCL reconstruction do not appear to have important effects on the biomechanical characteristics of the reconstructed knee [37], hence it was not the focus point in determining tibial tunnel placement in our study. Instead, we paid more attention to the placement of the tibial tunnel in the proximal-distal direction with reference to the tibial plateau and fibular head.

When taking measurements, we chose to use the lateral knee radiographic view, as this has been found to be the most clinically pertinent intraoperative radiographic measurement [32] and is commonly used to aid in the placement of the transtibial tunnel during $P C L$ reconstruction. Taking reference from the superior tip of the fibular head in our measurements was a natural choice as this is a more consistent bony landmark. In totality, the effect of these choices provided assurance that the recorded measurements are both objective and reproducible.

We understand that the tibial tunnel route is in a 3-dimensional structure, and identifying it based on just one 2-dimensional image (single lateral radiograph of the knee) is difficult and illusive. However, as it is not easy to accurately identify the tibial tunnel exit point on the AP radiograph, and for the same reasons stated above (regarding the mediallateral placement of the tibial tunnel not being as crucial), it was not assessed in this study. This was a retrospective study and our patients did not routinely have any other radiological investigations (either CT or MRI scans) performed postoperatively. We are looking to include these in future prospective studies to allow us to better identify the tibial exit point in a 3-dimensional way instead of the current 2-dimensional method using a single lateral radiograph.
Our data shows that there is a strong negative correlation between the actual distance of the tibial tunnel from the superior tip of fibular (FTL) and improvement in Lysholm scores (Figure 4). This suggests that there would be better outcomes if the tunnel is closer to the tip of the fibula. In our study, we had 12 out of 30 patients with tibial tunnels > $15 \mathrm{~mm}$ from the tibial plateau, 15 out of 30 of our patients with tibial tunnels within $10-15 \mathrm{~mm}$ below the tibial plateau, as suggested by current studies [8,36-38], and 3 out of 30 patients having tibial tunnels that were $<10 \mathrm{~mm}$ from the tibial plateau.

Although our findings may be helpful, we would like to highlight several limitations. The most significant limitation is the small sample size. This is inadvertent as PCL injuries are recognized to be infrequent and may not be symptomatic enough to require surgical intervention [38]. Larger studies involving more patients would be helpful in supporting our findings and confirming the correlations found. Next, our study is also limited in the evaluation of clinical outcomes. We focused on Lysholm scores because it was the only measure where data was retrospectively available for all patients. We acknowledge the value of other subjective and objective scoring schemes and encourage a combined use of multiple schemes in future studies as a means to achieve a more comprehensive perspective of the significance of tibial tunnel placement. Finally, we were not able to evaluate the femoral tunnel placement in our study that was based solely on post-operative radiographs of the knee, resulting in a potential source of confounding bias. However, this was a single surgeon study, with the femoral tunnel position being kept as constant as possible, in order to reduce the degree of bias.

\section{Conclusion}

In this paper, we present our findings of a strong correlation observed between distance of tibial tunnel to superior tip of fibula (FTL) and improvement in Lysholm scores. This can potentially be used to predict the improvement in subjective outcome scores of patients (post-PCL reconstruction), based on tibial tunnel placement. The previously recommended placement of tibial tunnel $10-15 \mathrm{~mm}$ below the tibial plateau is a good recommendation, but placement of this tunnel > $15 \mathrm{~mm}$ appears to trend towards better outcome scores. Our method can be used as a reliable guide for satisfactory placement of the tibial tunnel for PCL surgery intra-operatively and the prediction of post-operative outcome scores.

\section{Conflict of Interests}

The author(s) declared no potential conflicts of interest with respect to the research, authorship, and/or publication of this article.

\section{Funding}

The author(s) received no financial support for the research, authorship, and/or publication of this article.

\section{References}

1. Miyasaka K (1991) The incidence of knee ligament injuries in the general population. American Journal of Knee Surgery 1: 43-48. 
2. Zayni R, Hager JP, Archbold P, et al. (2011) Activity level recovery after arthroscopic $\mathrm{PCL}$ reconstruction: A series of 21 patients with a mean follow-up of 29 months. Knee 18: 392-395.

3. Stannard JP, McKean RM (2009) Anatomic PCL reconstruction: The double bundle inlay technique. Operative Techniques in Sports Medicine 17: 148-155.

4. Voos JE, Mauro CS, Wente T, et al. (2012) Posterior cruciate ligament: Anatomy, biomechanics, and outcomes. Am J Sports Med 40: 222-231.

5. Moorman CT, Zane MSM, Bansai S, et al. (2008) Tibial insertion of the posterior cruciate ligament: A sagittal plane analysis using gross, histologic, and radiographic methods. Arthroscopy 24: 269-275.

6. Nicodeme JD, Löcherbach C, Jolles BM (2014) Tibial tunnel placement in posterior cruciate ligament reconstruction: A systematic review. Knee Surg Sports Traumatol Arthrosc 22: 1556-1562.

7. Chia S, Kapoor V, Ali M, et al. (2002) The posterior cruciate ligament: An anthropometric study in Asians and evaluation of safe limits for bony tunnel creation during reconstruction. Ann Acad Med Singapore 31: 631-635.

8. Vaquero-Picado A, Rodríguez-Merchán EC (2017) Isolated posterior cruciate ligament tears: An update of management. EFORT Open Rev 2: 89-96.

9. Gali JC, Esquerdo P, Almagro MAP, et al. (2015) Radiographic study on the tibial insertion of the posterior cruciate ligament. Rev Bras Ortop 50: 342-347.

10. Teng $Y$, Guo L, Wu M, et al. (2016) MRI analysis of tibial PCL attachment in a large population of adult patients: Reference data for anatomic PCL reconstruction. BMC Musculoskelet Disord 17: 384.

11. Lind M, Nielsen TG, Behrndtz K (2018) Both isolated and multiligament posterior cruciate ligament reconstruction results in improved subjective outcome: Results from the danish knee ligament reconstruction registry. Knee Surg Sports Traumatol Arthrosc 26: 1190-1196.

12. Covey D, Sapega AA, Sherman GM (1996) Testing for isometry during reconstruction of the posterior cruciate ligament: Anatomic and biomechanical considerations. Am J Sports Med 24: 740-746.

13. Kim Y-M, Lee CA, Matava MJ (2011) Clinical results of arthroscopic single-bundle transtibial posterior cruciate ligament reconstruction: A systematic review. Am J Sports Med 39: 425-434.

14. Lahner M, Vogel T, Schulz M, et al. (2012) Outcome 4 years after isolated single-bundle posterior cruciate ligament reconstruction. Orthopade 41: 206-211.

15. Lipscomb AB, Anderson AF, Norwig ED, et al. (1993) Isolated posterior cruciate ligament reconstruction: Long-term results. Am J Sports Med 21: 490-496.

16. Qi Y-S, Wang H-J, Wang S-J, et al. (2016) A systematic review of double-bundle versus single-bundle posterior cruciate ligament reconstruction. BMC Musculoskelet Disord 17: 45.

17. Harner CD, Höher J (1998) Evaluation and treatment of posterior cruciate ligament injuries. Am J Sports Med 26: 471-482.

18. Race A, Amis AA (1998) PCL reconstruction. In vitro biomechanical comparison of "isometric" versus single and double-bundle 'anatomic' grafts. J Bone Joint Surg Br 80: 173-179.

19. Galloway MT, Grood ES, Mehalik JN, et al. (1996) Posterior cruciate ligament reconstruction: An in vitro study of femoral and tibial graft placement. Am J Sports Med 24: 437-445.

20. Jeong WS, Yoo YS, Kim DY, et al. (2010) An analysis of the posterior cruciate ligament isometric position using an in vivo 3-dimensional computed tomography-based knee joint model. Arthroscopy 26: 1333-1339.

21. Trus P, Peterman J, Gotzen L (1994) Posterior cruciate ligament $(\mathrm{PCL})$ reconstruction - an in vitro study of isometry. Knee Surgery, Sports Traumatology, Arthroscopy 2: 100-103.

22. Grood ES, Hefzy MS, Lindenfield TN (1989) Factors affecting the region of most isometric femoral attachments: Part I: The posterior cruciate ligament. Am J Sports Med 17: 197-207.

23. Juergensen K, Edwards J, Jakob R (1994) Positioning of the posterior cruciate ligament. Knee Surgery, Sports Traumatology, Arthroscopy 2: 133-137.

24. Sidles JA, Larson RV, Garbini JL, et al. (1988) Ligament length relationships in the moving knee. J Orthop Res 6: 593-610.

25. Bomberg B, Acker J, Boyle J, et al. (1990) The effect of posterior cruciate ligament loss and reconstruction on the knee. The American Journal of Knee Surgery 3: 85-96.

26. Bach BR, Daluga DJ, Mikosz R, et al. (1992) Force displacement characteristics of the posterior cruciate ligament. Am J Sports Med 20: 67-72.

27. Friederich N, O'Brien W (1992) Functional anatomy of the cruciate ligaments. The Knee and the Cruciate Ligaments, 78-91.

28. Kokron AE, Hernandez AJ, Camanho GL, et al. (2001) Isometry of the posterior cruciate ligament. Acta Ortopédica Brasileira 9: 49-52.

29. Racanelli JA, Drez D (1994) Posterior cruciate ligament tibial attachment anatomy and radiographic landmarks for tibial tunnel placement in PCL reconstruction. Arthroscopy: The Journal of Arthroscopic \& Related Surgery 10: 546-549.

30. Girgis FG, Marshall JL, Jem AAM (1975) The cruciate ligaments of the knee joint. Anatomical, functional and experimental analysis. Clin Orthop Relat Res 106: 216-231.

31. Ramos LA, de Carvalho RT, Cohen M, et al. (2008) Anatomic relation between the posterior cruciate ligament and the joint capsule. Arthroscopy 24: 1367-1372.

32. Johannsen AM, Anderson CJ, Wijdicks CA, et al. (2013) Radiographic landmarks for tunnel positioning in posterior cruciate ligament reconstructions. Am J Sports Med 41: 35-42.

33. Tompkins M, Keller TC, Milewski MD, et al. (2014) Transtibial tunnel placement in posterior cruciate ligament reconstruction. Orthop J Sports Med 2.

34. Van Tongel A, MacDonald PB (2010) Single bundle posterior cruciate ligament reconstruction: Surgical technique and results. Sports Med Arthrosc Rev 18: 238-241.

35. Sekiya JK, West RV, Ong BC, et al. (2005) Clinical outcomes after isolated arthroscopic single-bundle posterior cruciate ligament reconstruction. Arthroscopy 21: 1042-1050.

36. Shin YS, Han SB, Hwang YK, et al. (2015) Tibial tunnel aperture location during single-bundle posterior cruciate ligament reconstruction: Comparison of tibial guide positions. Arthroscopy 31: 874-881.

37. Markolf KL, McAllister DR, Young CR, et al. (2003) Biomechanical effects of medial-lateral tibial tunnel placement in posterior cruciate ligament reconstruction. J Orthop Res 21: 177-182.

38. Rosenthal MD, Rainey CE, Tognoni A, et al. (2012) Evaluation and management of posterior cruciate ligament injuries. Phys Ther Sport 13: 196-208. 